\title{
Erythropoietin Nanopariticles: Therapy for Cerebral Ischemic Injury and Metabolize in Kidney
}

\author{
Ting Wang 1,a, Yan $\mathrm{Hu}^{2}$, Long Zhang², Li Jiang², Nongyue He ${ }^{1^{*}}$ \\ 1State key Laboratory of Bioelectronics, Southeast University, Nanjing 210096, China \\ ${ }^{2}$ Teaching and Research Section of Pediatrics, Clinical Medical College, Southeast University, Nanjing 210009, China \\ a Present address: 930 N. University Ave, room 4624, Ann Arbor, MI, USA. 48109, E-mail: wangting@umich.edu \\ *Corresponding authors. Email: nyhe@seu.edu.cn
}

\begin{abstract}
Ischemic metabolism in the enterogram is a crucial issue of clinical concern for deep understanding of drug delivery. In this study we use nanochemistry in micelle to produce the nanocarrier of erythropoietin (EPO) and traced the metabolizable process of the EPO carrier in vivo. Nascence Sprague-Dawley rats of 5 days are treated with EPO-loading nanocarriers. Curative effects of the EPO nanocarriers for periventricular leukomalacia (PVL) models are validated by the analysis of pathology and praxiology of the mice. We demonstrate in vivo that EPO nanocarriers can ameliorate drug-induced liquefaction caused by hypoxia. By tracking the metabolism of EPO in liver and kidney, we suggest that nanocarriers effectively prolong the metabolic half-life and clearance time of EPO. HPLC results show that exciting amino acid toxicity was inhibited, since mobilization of late oligodendrocyte can be protected by treatment of EPO vehicles from hypoxia.
\end{abstract}

Keywords: Brain hypoxia; Nanoparticles; Erythropoietin; Oligodendrocyte

Citation: T. Wang, et al. Erythropoietin Nanopariticles: Therapy for Cerebral Ischemic Injury and Metabolize in Kidney. Nano Biomed Eng. 2010, 2(1), 31-39. DOI: 10.5101/nbe.v2i1.p31-39.

\section{Introduction}

Erythropoietin (EPO) is a type of glycoprotein hormone secreted by the kidney whose primary function is to stimulate the bone marrow to enhance red blood cell production. EPO has been widely used in anemia cure to increase the hemoglobin of patients with ischemia and hypoxia who become anemic secondary to their disease [1-3]. As a prime regulator of red blood cell production in addition to its indirect effect to increase the oxygen-carrying capacity of blood, it was reasonable to postulate that EPO carriers have beneficial effects on brain hypoxia [4], although mechanisms of cerebral palsy and stuntedness are not fully understood.

EPO is mainly produced in the renal interstitium in response to hypoxic stimulate. [5] The direct injection of EPO cannot avoid the damage of the protease. For this reason, large quantities of EPO are always needed in therapy. Over quantity injection of the synthetic version of EPO risks the safety of the user. By increasing the thickness of the blood, EPO increases the risk of blood clotting which can block blood vessels causing a heart attack or stroke. This hormone is produced synthetically to treat patients with kidney failure, cancer, and AIDS. Large quantity of EPO also causes hypertension, and can lead to seizures and congestive heart failure [6-8]. We demonstrate from our experiment that drop with chitosan-alginate(CS/AL) nanoparticles carrier, small quantity of EPO nanocarriers have the equal effect as large quantity of EPO that was directly injected on therapy of ischaemia injury. Our results deduce that with the protection of CS/AL nanoparticles, EPO can avoid the damage from the protease and enhance the effecting of therapy. In the model of postnatal day 5 (P5) rats which were injected with 3-Nitroproprionic 
nanobe.org

Acid (3-NP), we found the increase of both glial cells and expression of proin-flammatory cytokines occurred with hypoxic stimulate. However, injection of EPOdoped nanoparticles significantly ameliorated liquefaction caused by drug-induced hypoxia. EPOdoped nanocarriers are spherical, highly monodispersed, and stable in aqueous system. We analyzed the effects of EPO carriers on the total number of circulating hematopoietic progenitor cells (cHPCs) before and at indicated time points during EPO therapy.

In conclusion, protective effect of EPO-doped nanoparticles against anoxemia with small quantities has been stressed in the kidney, neural tissue and application of this extra hematopoietic effect clinically, however, large numbers of or excessive EPO has been associated with undesirable side effects, such as thrombotic events and we describe above [9-11]. Thus, the development of a nanocarrier of erythropoietin has been desirable.

\section{Materials and Methods}

\subsection{3-NP injure}

Based on our previously established method [12], every injured rat was encephalic injected with $1 \mu \mathrm{L}$ of 3-NP (Sigma, $\mathrm{n}=10$ ) in $300 \mathrm{mM}$ of PBS (Sinopharm Chemical Reagent Co. Ltd) with stereotaxic guidance. Anaesthesia was introduced by injecting fentanyl citrate sub-cutaneously at a dose of $0.3 \mu \mathrm{g} / \mathrm{kg}$ and fluanisine (Hypnorm, Janssen) at $10 \mu \mathrm{g} / \mathrm{kg}$. The forelimb motor cortex (MC) was exposed. With stereotaxic guidance, a glass tip attached to a Hamilton syringe was deployed $1.0 \mathrm{~mm}$ posterior to the bregma, $1.0 \mathrm{~mm}$ lateral to the midline, and $2.0 \mathrm{~mm}$ below the skull surface. The needles were kept at these points for 5 minutes and then slowly removed out of the brain. EPO nanoparticles were injected subcutaneously, with a concentration of $200 \mathrm{IU} / \mathrm{kg}$. The blank group was treated in the same manner. The wound was sutured after the procedure. Anaesthesia was reversed while maintaining analgesia by an intraperitoneal injection of pentobarbital sodium $(1 \mu \mathrm{g} / \mathrm{kg})$. All rats survived after surgery.

\subsection{Preparation of EPO encapsulations}

EPO nanoparticles were obtained by ionic gelation of sodium alginate (AL) (Sigma) and chitosan (CS) (Mw. 10000, degree of deacetylation $>88 \%$ ) which is a commercial product of Haidebei Halobios Co., Ltd. (Ji'nan, China), based on one of our related work [13]. EPO commercial products (Sunshine pharmaceutical Co. Ltd. Shenyang, China) 200IU were mixed with a 3wt\% aqueous solution of chitosan. EPO nanoparticles were obtained upon the addition of AL solution into chitosan microemulsion solution keeping ratio of AL/CS to be 2 to 5 under mild mechanical stirring (800 $\mathrm{rpm}$ ) at room temperature. Acetic acid was used to control the $\mathrm{pH}$ below 6.5 during reaction. The EPO nanoparticles were collected by centrifuge at 4000 $\mathrm{r} / \mathrm{min}$ and freeze-dried after washed three times with PBS. The surface morphology of nanoparticles was characterized by scanning electron micro-scope (SEM) (JEOL JSM-T 220A scanning electron micro-scope, JEOL Ltd, Japan) operating at an accelerating voltage of $10-30 \mathrm{kV}$ without sputtering with gold.

EPO-loading nanoparticles were deliquesced in citrate to check the drug loading amount and the drug release. Enzyme calibration (SLT-210, COM. DYNEX U.S.A) and EPO kit (Pharmaceutical Factory of Shanghai, China) were used to detect the amount of EPO that releasing from nanocapsules. The drugloading (DL) ratio of EPO can be obtained from the formula below:

$$
\mathrm{DL} \%=\mathrm{W}_{\mathrm{a}} / \mathrm{W}_{\mathrm{t}} \times 100
$$

(Wa: amount of EPO in nanoparticles, Wt: total mass of nanoparticles.)

In the release measurement, phosphate buffered saline (PBS) solution ( $\mathrm{pH}=7.4$ ) was used as the release medium. EPO-loading capsules were immerged in PBS solution at velocity of $100 \mathrm{r} / \mathrm{min}$ at $37^{\circ} \mathrm{C}$ for $600 \mathrm{~h}$. DL was tested at the points of every $8 \mathrm{~h}$ by extracting $5 \mathrm{~mL}$ of the EPO solution while adding the same volume of PBS to keep a constant volume. The release-time curve was obtained based on the readings from enzyme calibration.

\subsection{EPO-loading nanoparticles cure}

32 postnatal days 5 (P5) rats were selected from the Clinical Medicine School of the Southeast University, they were divided randomly into 3 groups: (1) injury group, encephalic injected with 3-NP then intraperitoneally injected with blank nanoparticles; (2) therapy group, after encephalic injected with 3-NP, offered with therapy by EPO-droped nanoparticles celiac injection; (3) Control group, just treated by PBS.

\subsection{Metabolism of EPO-loading nanoparticles}

Metabolism of EPO-loading nanoparticles was demonstrated by 3-NP injured rats that were treated with EPO-loading nanoparticles loaded with fluorescein isothiocyanate (FITC). Mice were fed normally and sacrificed at 8 hours, 24 hours, 36 hours and 48 hours. Under nembutal anesthesia (40 mg/kg), liver and kidney were obtained. The suspended liver and kidney were fixed by deep frozen (liquid nitrogen) clamp. The frozen tissue was slice into pieces of $30 \mu \mathrm{m}$ thick by Leica CM1850 cryostat.

\subsection{Myelin basic protein (MBP) analysis}

The analysis of immature oligodendrocytes was based on indirect immunofluorescent assay. The frozen brain slices of $72 \mathrm{~h}$ at $20 \mu \mathrm{m}$ thickness were immersed in an antigen against immature oligodendrocytes 
nanobe.org

antibodies IN-1 $(10 \mu \mathrm{m} / \mathrm{mL})$ at $4^{\circ} \mathrm{C}$ overnight. After the sections were rinsed twice in $0.01 \mathrm{M}$ PBS for $5 \mathrm{~min}$, they were banded with antibodies IN-1. The primary antibodies IN-1 on the sections were marked with the secondary FITC antibodies in a darkroom for 1 hour at $37^{\circ} \mathrm{C}$. The white matter around the ventricle was observed under a fluorescence microscope. The damage of PVL was checked by detecting the averaged amount of oligodendrocytes in 6 fields of view, each sample having 3 frozen slices.

\subsection{Microdialysis experiments}

Microdialysis cannulas $2 \mathrm{~mm}$ long and $0.5 \mathrm{~mm}$ in diameter (CMA12; Bioanalytical Systems, West Lafayette, IN, U.S.A.) were used. Before implantation, each probe was flushed with distilled water for $5 \mathrm{~min}$ at a flow rate of $40 \mu \mathrm{l} / \mathrm{min}$, then simulated body fluid (SBF) was used in same way. Sprague-Dawley (SD) rats were anesthetized and placed in a stereotaxic frame as described above. Animals were implanted unilaterally with a microdialysis cannula. Probes were deployed $1.0 \mathrm{~mm}$ posterior to the bregma, $1.0 \mathrm{~mm}$ lateral to the midline, and $2.0 \mathrm{~mm}$ below the skull surface. Animals maintained under low anesthesia (0.5\% halothane) throughout the experiment. The probes were perfused continuously with the SBF at a flux rate of $2 \mu \mathrm{l} / \mathrm{min}$ using a microinjection pump (Carnegie CMA 100). After a 60-min equilibration period, 25- $\mu$ l (12.5-min) consecutive fractions of perfusate were continuously collected and analyzed by high-performance liquid chromatography (HPLC) as described below.

\subsection{Amino acid analysis of striatal and hippocampal dialysates}

Amino acids were measured in dialysates by HPLC as previously described [14]. In brief, the 25- $\mu$ l collected fractions were derivatized with the same volume of o-phthalaldehyde and 3 min later $20 \mu \mathrm{l}$ was injected into an HPLC system (Beckman). An ODS column (25 cm $\times 4 \mathrm{~mm}$ internal diameter) was used. The mobile phase was methanol/potassium acetate $(0.1$ $\mathrm{M}, \mathrm{pH} 5.5$ ) and was run at a rate of $1.5 \mathrm{ml} / \mathrm{min}$ in a linear gradient (50-min duration) from 25 to $75 \%$ methanol. The results obtained were compared with a standard mixture of amino acids (Sigma) equally processed.

\section{Results and discussion \\ 3.1 EPO-loading nanoparticles}

EPO-loading nanoparticles with spherical-shape own a network associated through electrostatic interactions from chitosan and AL. EPO loaded particles possess similar size distribution and monodispersion as shown in Fig. 1. The diameter of the particles is around 400 $\mathrm{nm}$. To investigate the stability of release, the release rate of EPO was observed during $530 \mathrm{~h}$ in PBS medium $(\mathrm{pH}=7.4)$ and the diffused concentration-time curve was shown in Fig. 2. Release time for EPO that released from nanoparticles in $\mathrm{PBS}(\mathrm{pH}=7.4)$ solution and in blood stream is similar because of the same $\mathrm{pH}$.

Chitosan is one of the most important compositions for drug vehicle, which is almost completely composed of glucose pyranoside in the microcapsules and is not completely deacetylated. Therefore, the crosslinking density of the polyelectrolyte was reinforced when the amino was ionized into amido in the solution under the condition of $\mathrm{pH}<7.0$, which increased the compaction of the shell. However, in the PBS ( $\mathrm{pH}=7.4)$, alkaline of the solution decreased the amount of cationic amido, which decrease the crosslinking density of the polyelectrolyte complexes produced, and brought about the incompactness of shell and the release of EPO from CS/AL nanoparticles.

Fig. 2 shows that the EPO releasing from the nanocapsules was slowly increasing in the first 24 hour, then the curve became gentle after that and almost kept in line during 313h. After 313h the concentration dropped down as show in the curve. The releasing curve can be attributed to two factors: first, the degradation of nanoparticles was the reason for instantaneous increase of spectrum; second, slow penetration of PBS into the shell brought a slowly change in cross-linking density of the polyelectrolyte complexes, leading to a slow releasing of EPO after the abrupt increase of the signal [15-17].

The results demonstrated that nanoparticles can effectively control the drug release during $500 \mathrm{~h}$. 
Figure 1. SEM image for EPO-loading nanoparticles.

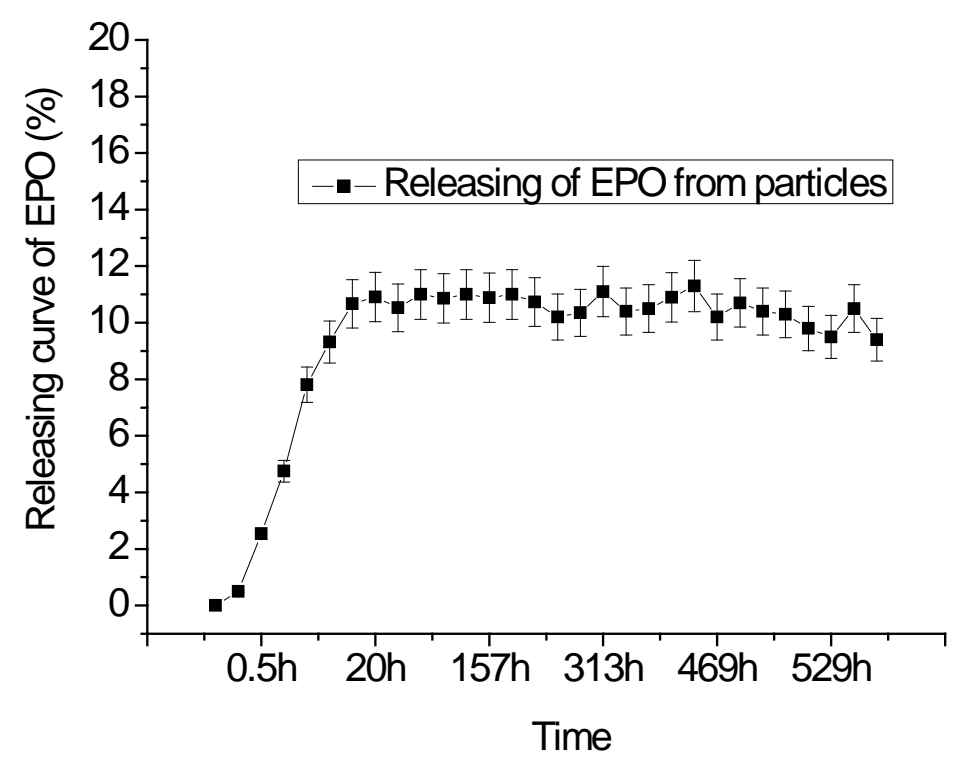

Figure 2. EPO releasing curve in 529h.

\subsection{EPO cures and metabolize of EPO nanoparticles}

\section{in vivo}

As Using a flourasence dye particles to demonstrate the EPO loaded particles activity in vivo is a novel and useful experimental tool for assessing particle function in kiney where EPO expression levels is unknown. It should prove particularly useful in investigating the effects of altered body metabolism of drugs or endogenous neurochemicals on drug therapeutic efficacy, drug interactions, neurotoxicity, and behavior. Selective induction or inhibition of metabolically active 
nanobe.org

drug metabolizing enzymes in the body may also provide ways to control drug activation in specific brain regions as a novel therapeutic avenue [18].

Newborn rats are more sensitive to EPO than adult because their kidneys are less developed and unable to metabolize and excrete EPO. The one with 3-NP injure may be more sensitive to EPO effects, especially those with compromised kidney functioning. All of the blood that leaves the liver and intestines must pass through the kidney before been clear. Thus, the kidney is strategically placed to process nutrients and drugs absorbed from the digestive tract into forms that are easier for the rest of the body to use. We found that the
EPO carried particles were not be found in kidney at the first $4 \mathrm{~h}$ and completely cleared until $72 \mathrm{~h}$ (Fig. 3). The study of erythropoietin monomer shown that EPO is metabolized completely in $12 \mathrm{~h}$ (Erythropoietin protects neurons against chemical hypoxia and cerebral ischemic injury by up regulating $\mathrm{Bclx}(\mathrm{L})$ expression) and peak value of EPO monomer in blood will be on 8 h after injection. From our study, EPO nanoparticles were kept in liver with consistent value during $24 \mathrm{~h}$ and in vivo in $36 \mathrm{~h}$, after injection. The results showed that with the drug-loading vehicles, EPO can stay in vivo longer and in this way the therapy effect of EPO will be enhanced definitely.
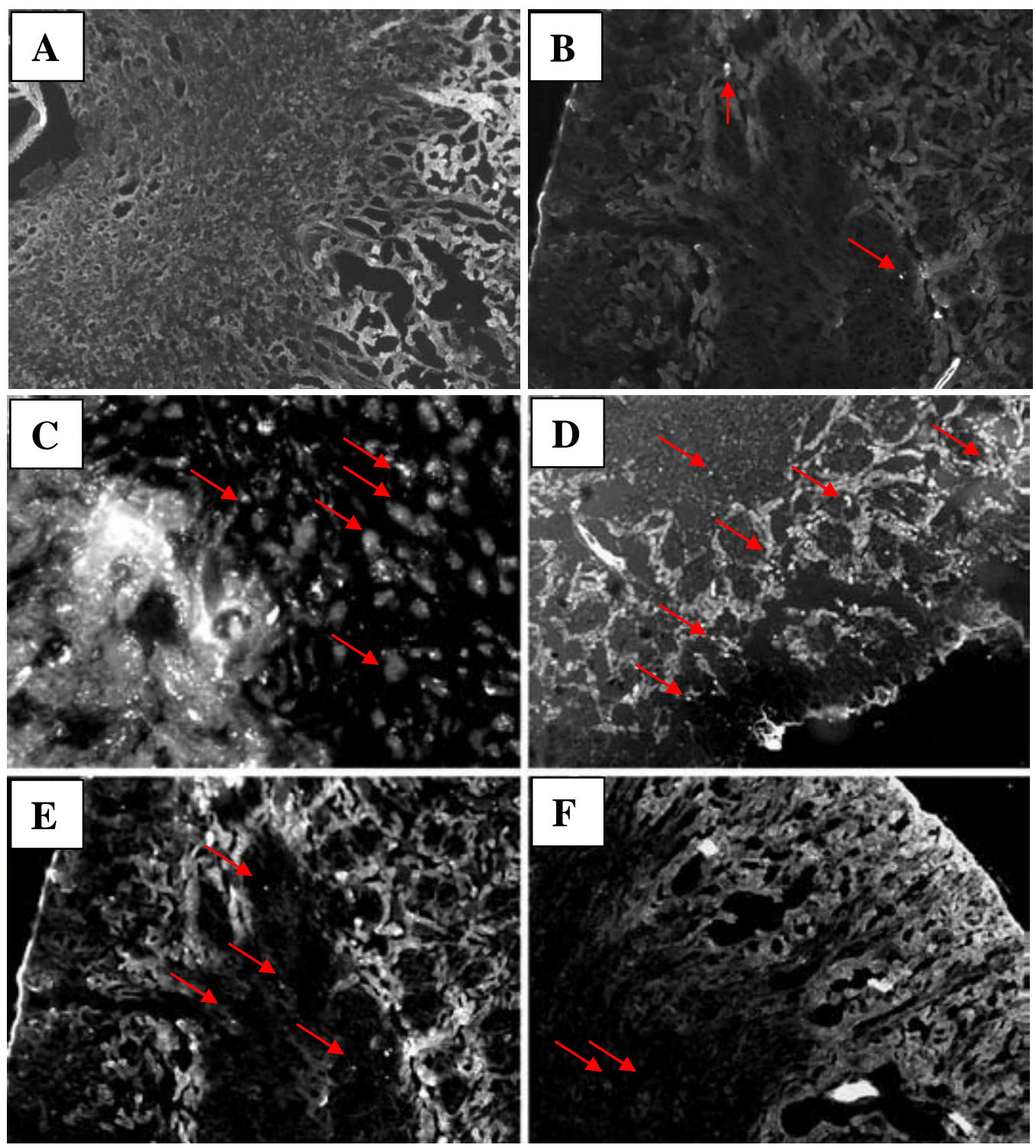

Figure 3. Metabolism of the particles in kidney. (A) Nanoparticles can not be found in kidney, (B) 8 h after intraperitoneally injected EPO-NP, quantity of EPO-NP concentrated in renalvein of kidney (C) EPO-NP concentrated in glomerulus, after $12 \mathrm{~h}$ of intraperitoneal injection, (D) $24 \mathrm{~h}$ after intraperitoneal injection nanoparticles can be found in calyces renales minores (E) $48 \mathrm{~h}$ after intraperitoneal injection the small quantity of fluorescence particles can be found in pelvis. (F) After $72 \mathrm{~h}$ few nanopartcles can be found leave in pelvis. 


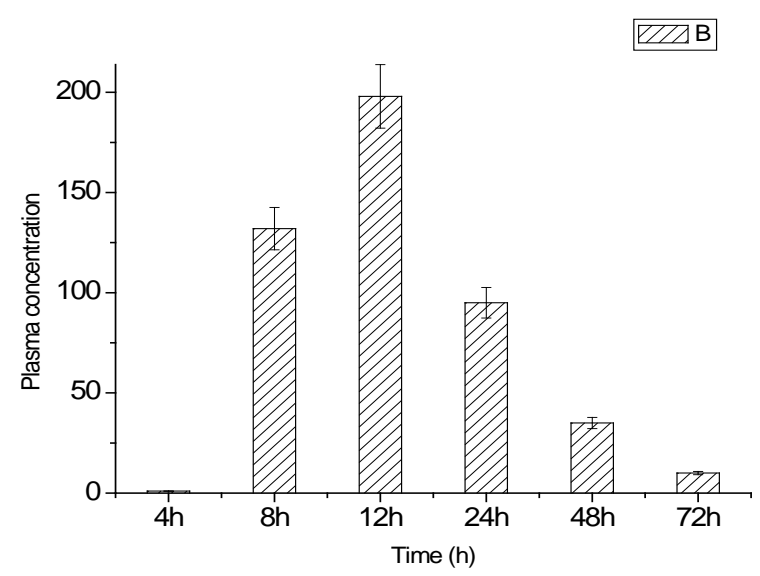

Figure 4. Plasma concentration of EPO-loaded nanoparticles in experimental group

Metabolism of EPO has been proved that in the kidney, liver, heart and neural tissue [18]. Fig. 4 shows that in kidney, FITC loaded particles metabolized and been cleared in the kidney. We found from transverse section of kidney that in the first $8 \mathrm{~h}$ the nanoparticles were accumulated in the renal corpuscle and renal calyx. During $12 \mathrm{~h}$ to $24 \mathrm{~h}$ a number of nanoparticles dispersed in pyramid, cone and the renal cortex, then in $48 \mathrm{~h}$ less particles dispersed in cone and the renal cortex. After $48 \mathrm{~h}$ the particles almost metabolized completely. The quantities of fluorescence radiation from EPO-loaded nanoparticles show the same result (Fig 4).

\subsection{MBP analyse}

Decrease in the amount of positive cell is attributed to the infection caused by 3-NP through increased lipid peroxidation, nitrite concentration, depleted superoxide dismutase activity and catalase enzyme activity in striatum [19].
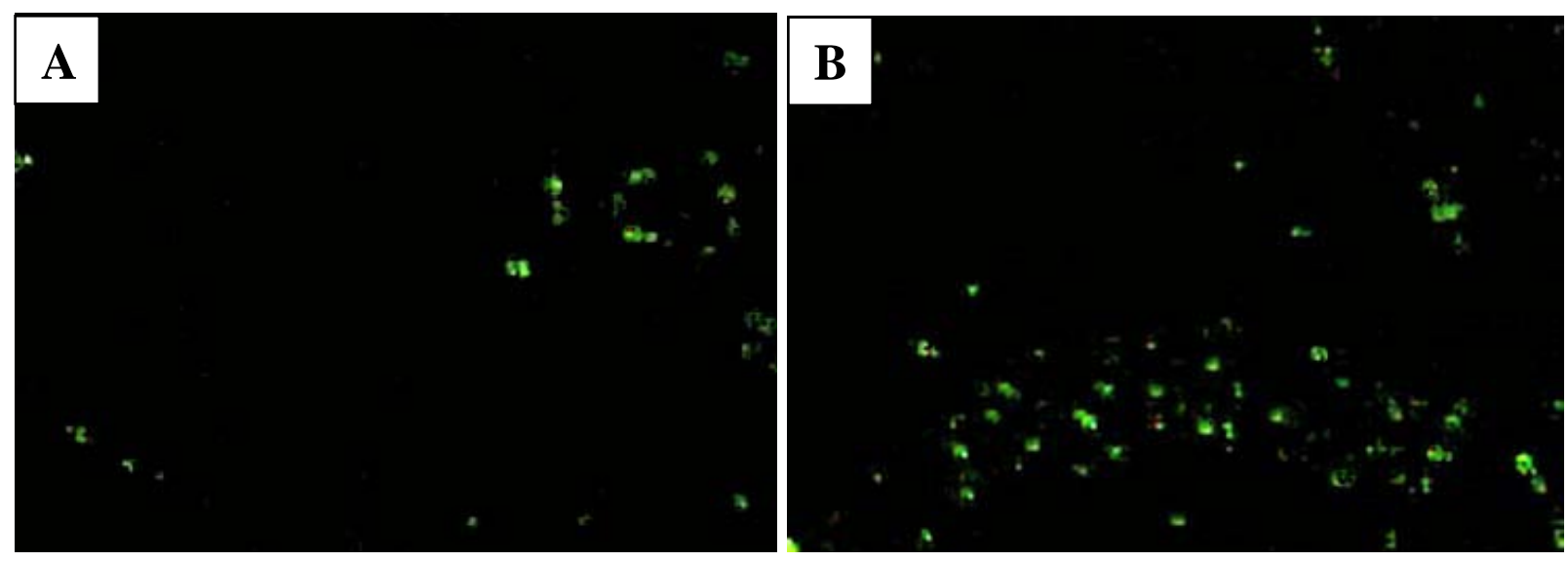

Figure 5. MBP positive cell image for (A) injured group and (B) EPO treated group on P5 ( \pm \pm ,,$n=6)$.

As compared to EPO carrier treated group statistical analyses of average optical density showed obvious that damage to positive cells are stronger in injured group due to 3-NP attack than therapy group injected with EPO nanocarrier (Fig. 5). Pearson correlation coefficient $\mathrm{P}<0.05$ was found. EPO nanoparticles treatment significantly attenuated lipid peroxidation, nitrite concentration; restored superoxide dismutase activity and catalase enzyme activities prevent the decrease from immature OLs and myelin basic proteins. EPO therapy effectively protected the growth of myelin from the 3-NP damage, characteristics of recovery of brain injury was significant.

\subsection{HPLC analyse}

After 3-NP injection, succinic dehydro- genase was inhibited, amino acids such as Glu accumulated in the brain slice. The prolonged activation of excitatory amino acids lead to excitotoxicity to oligoden- droglial 
nanobe.org

cell and thus causes white matter damage and tissue anoxia [20]. HPLC analyze shown that (Fig. 6) after injection of 3-NP, the signal from exciting amino acid are very strong. The HPLC signal of several types of excitatory amino acids: glutamic acid, asparthione, acetylcholine and dopamine can be found in HPLC image. The key reason for this excitotoxicity is because glutamate can increase the concentration of intracellular $\mathrm{Ca}^{2+}\left(\left[\mathrm{Ca}^{2+}\right]\right)$ in neurons, and thus finally induce neuronal death by activating proteases, phospholipases, and endonucleases. Several mechanisms explain how glutamate increases intracellular $\mathrm{Ca}^{2+}$ level. The activation of $\mathrm{Ca}^{2+}$. permeable N-methyl-D-aspartate (NMDA) receptors, opening of voltage-dependent $\mathrm{Ca}^{2+}$ channels following membrane depolarization induced by activation of 2amino-3-hydroxy-5-methyl-4-isoxazolepropionate (AMPA) receptors, and/or activation of metabotropic glutamate receptors (mGluRs) linked to phosphoinositide hydrolysis, which releases $\mathrm{Ca} 2+$ from intracellular stores, are leading factors. Glutamate is the principal excitatory neurotransmitter. After mediated by EPO nanoparticles treatment, exciting amino acid decreased significantly. Signal from inhibitory neurotransmitter glycin sign with red circle increased after the therapy from $36 \mathrm{~h}$ to $48 \mathrm{~h}$.

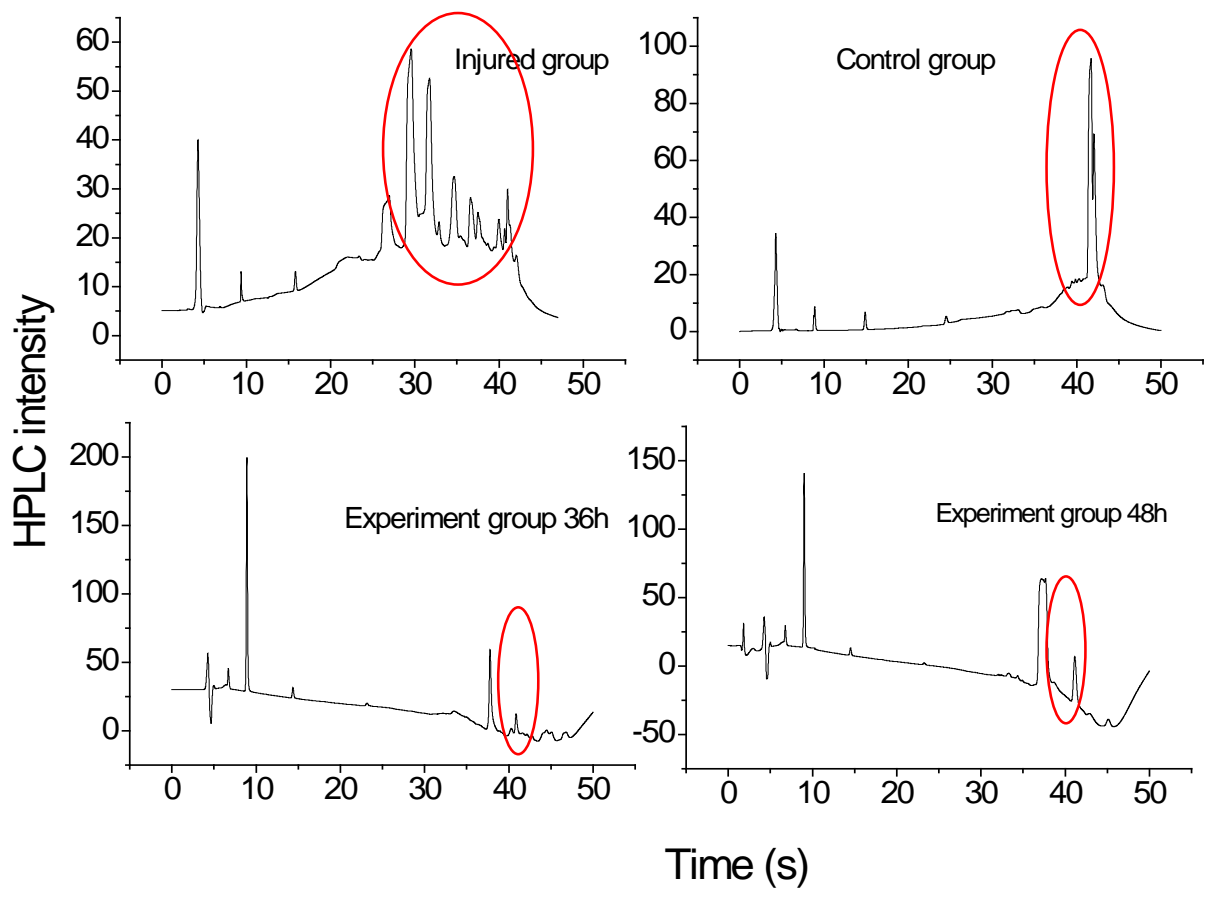

Figure 6. HPLC intensity shows us the change of amino acid

\section{Conclusion}

Particle science has been at the forefront of nanotechnology and fine-particle such as the one which has special structure and good biocompability since the early 1990s. Nowadays nanoparticles are used in broad fields such as targeted drug delivery, biomedical applications, tissue engineering, food and cosmetics [21-24].

In our work EPO nanoparticles were used to treat ischemic injury from 3-NP neurotoxin damage. The results demonstrate that EPO-loading nanoparticles' can protect decrease from immature OLs and myelin basic proteins by: (1) Prolonging plasma half-life of EPO; (2) Slowing metabolism decreasing the excitatory amino acids expression, and elevating the inhibitory neurotransmitter expression; (3) Reducing of excitotoxicity expression leads to survival of immature OLs cells; (4) enough quantity of immature OLs cells advance the growth of myelin; (5) Myelin formation and growth help brain recover from ischemic injury from 3-NP.

EPO-loading nanoparticles have intrinsic properties to preferentially accumulate in liver and kidney while sparing healthy for normal cells. Some wonderful characters of this kind of nanoparticles such as biodegradable, non-toxicity, high encapsulation and low releasing all provide a new therapeutic strategy in disease treatment.

\section{Acknowledgements}

We gratefully acknowledge the support to this research from the National Key Program for Developing Basic 
nanobe.org

Research (NO. 2007CB936104 and 2010CB933903),

Natural Science Foundation of China (NO. 60927001), and the 863 High Tech Project (NO. 2007AA022007), as well as the Scientific Research Foundation of Graduate School of Southeast University.

\section{References}

1. Kumral A, Gonenc S, Acikgoz O, Sonmez A, Genc K, Yilmaz O, Gokmen N, Duman N, Ozkan H. Erythropoietin increases glutathione peroxidase enzyme activity and decreases lipid peroxidation levels in hypoxic-ischemic brain injury in neonatal rats. Biol Neonate 2005; 87: 1518. doi:10.1159/000080490

2. Kumral A, Ozer E, Yilmaz O, Akhisaroglu M, Gokmen N, Duman N, Ulukus C, Genc S, Ozkan H. Erythropoietin protects against necrotizing enterocolitis of newborn rats by the inhibiting nitric oxide formation. Biol Neonate 2003; 83: 224-228. doi:10.1159/000068926

3. Kumral A, Uysal N, Tugyan K, Sonmez A, Yilmaz O, Gokmen N, Kiray M, Genc S, Duman N, Koroglu TF, Ozkan H, Genc K. Erythropoietin improves long-term spatial memory deficits and brain injury following neonatal hypoxia-ischemia in rats. Behav Brain Res 2004; 153: 7786. doi:10.1016/j.bbr.2003.11.002

4. McClure MM, Threlkeld SW, Fitch RH. The effects of erythropoietin on auditory processing following neonatal hypoxic-ischemic injury. Brain Res. 2006; 1087: 190195. doi:10.1016/j.brainres.2006.03.016

5. Siren AL, Fratelli M, Brines M, Goemans C, Casagrande S, Lewczuk P, Keenan S, Gleiter C, Pasquali C, Capobianco A, Mennini T, Heumann R, Cerami A, Ehrenreich H, Ghezzi P. Erythropoietin prevents neuronal apoptosis after cerebral ischemia and metabolic stress. Proc Natl Acad Sci USA 2001; 98: 4044-4049. doi:10.1073/pnas.051606598

6. Calvillo L, Latini R, Kajstura J, Leri A, Anversa P, Ghezzi P, Salio M, Cerami A, Brines M. Recombinant human erythropoietin protects the myocardium from ischemiareperfusion injury and promotes beneficial remodeling. Proc Natl Acad Sci USA 2003; 100: 48024806. doi:10.1073/pnas.0630444100

7. Parsa CJ, Matsumoto A, Kim J, Riel RU, Pascal LS, Walton GB, Thompson RB, Petrofski JA, Annex BH, Stamler JS, Koch WJ. A novel protective effect of erythropoietin in the infarcted heart. J Clin Invest 2003; 112: 999-1007. doi:10.1172/JCI200318200

8. Bagul A, Hosgood SA, Kaushik M, et al. Effects of erythropoietin on ischaemia/reperfusion injury in a controlled nonheart beating donor kidney model. Transplant international, 2008; 21: 495-510. doi:10.1111 j.1432-2277.2007.00636.x

9. Spandou E, Papadopoulou Z, Soubasi V, Karkavelas G, Simeonidou C, Pazaiti A, Guiba-Tziampiri O. Erythropoietin prevents long-term sensorimotor deficits and brain injury following neonatal hypoxia-ischemia in rats. Brain Res. 2005; 1045: 22-30.

10. Wei L, Han BH, Li Y, Keogh CL, Holtzman DM, Yu SP. Cell Death Mechanism and Protective Effect of
Erythropoietin after Focal Ischemia in the Whisker-Barrel Cortex of Neonatal Rats. JPET 2006; 317: 109116. doi:10.1124/jpet.105.094391

11. Kellert BA, McPherson RJ, Juul SE. A comparison of highdose recombinant erythropoietin treatment regimens in brain-injured neonatal rats. Pediatric Res 2007; 61: 451455. doi:10.1203/pdr.0b013e3180332cec

12. Wang $\mathrm{T}$, Zhang, L, Jiang L, Nongyue He. Neurotoxicological effects of 3-nitropropionic acid on the neonatal rat. NeuroToxicol. 2008; 29: 1023-1029. doi:10.1 016/j.neuro.2008.07.006

13. Yang WJ, Wang T, He NY. Preparation and Property of Chitosan/Sodium Tripolyphosphate Microcapsules as Drug Carrier. Chem J Chinese U. 2009; 30: 625-628.

14. Salazar, J., Campbell M, Anderson S, Gardner S, Dunnum J. New records of Bolivian mammals. Mammalia 1994 ; 1 : 125-130. doi:10.1515/mamm.1994.58.1.125

15. Tozaki H, Odoriba T, Okada N, Fujita T, Terabe A, Suzuki T, Okabe S, Muranishi S, Yamamoto A. Use of protease inhibitors to improve calcitonin absorption from the small and large intestine in rats. J Pharm Sci. 1997; 86: 10161021. doi:10.1021/js970018g

16. Mahesh D. Chavanpatil, Ayman Khdair, Jayanth Panyam, Nanoparticles for Cellular Drug Delivery: Mechanisms and Factors Influencing Delivery. J. Nanosci. Nanotechnol 2006; 6: 2651-2663.

17. Kamat JP, Narurkar LM, Narurkar MV. Induction of hepatic drug-metabolizing enzymes by DL-methionine in rats. Journal of Biosciences 1989; 14: 233-241. doi:10. 1007/BF02716683

18. Esneault E, Pacary E, Eddi D, et al. Combined therapeutic strategy using erythropoietin and mesenchymal stem cells potentiates neurogenesis after transient focal cerebral ischemia in rats. J. Cerebral Blood Flow \& Metabolism 2008; 28: 1552-1563. doi:10.1038/jcbfm.2008.40

19. Perlman JM. Ola DS. Summary proceedings from the neurology group on Hypoxic-ischemic encephalopathy. Pediatrics 2006; 117: S28-S33.

20. Palmer C. Hypoxic-ischemic encephalopathy: Therapeutic approaches against microvascular injury, and role of neutrophils, PAF, and free radicals. Clinics in Perinatology 1995; 22: 481-517.

21. Bao CC, Yang H, Sheng P, Song H, Ding XH, Liu B, Lu YC, Hu GH, Cui DX. Cloning, Expression, Monoclonal Antibody Preparation of Human Gene NBEAL1 and Its Application in Targeted Imaging of Mouse Glioma. Nano Biomed Eng 2009; 1: 74-87.

22. Li H, Zhang YG, Huang WP. Photoactivation of Ionexchangeable Trititanate Nanotubes Modified by MS (M = Cd, Zn) Nanoparticles. Nano Biomed Eng 2009; 1: 48-56.

23. Cui DX, Han YD, Li ZM, Song H, Wang K, He R, Liu B, Liu HL, Bao CC, Huang P, Ruan J, Gao P, Yang H, Cho HS, Ren QS, Shi DL. Fluorescent Magnetic Nanoprobes for in vivo Targeted Imaging and Hyperthermia Therapy of Prostate Cancer. Nano Biomed Eng 2009; 1: 94-112.

24. Yang WJ, Wang T, Fu J, He NY, Chitosan/Sodium Tripolyphosphate Nanoparticles: Preparation, 
nanobe.org

Characterization and Application as Drug Carrier. J Biomed Nanotechnol. 2009; 5: 591-595. doi:10.1166/jbn.2009.1067

Received 20 January, 2010; accepted 18 February, 2010; published online 5 March, 2010.

Copyright: (c) $2010 \mathrm{~T}$. Wang et al. This is an open-access article distributed under the terms of the Creative Commons Attribution License, which permits unrestricted use, distribution, and reproduction in any medium, provided the original author and source are credited. 